\title{
The Impact of Background Error on Incomplete Observations for 4D-Var Data Assimilation with the FSU GSM
}

\author{
I. Michael Navon ${ }^{1}$, Dacian N. Daescu ${ }^{2}$, and Zhuo Liu ${ }^{1}$ \\ 1 School of Computational Science and Information Technology, \\ Florida State University, Tallahassee, FL \\ navon@csit.fsu.edu \\ 2 Dept. of Mathematics and Statistics, Portland State University, Portland, OR
}

\begin{abstract}
To assess the impact of incomplete observations on the $4 \mathrm{D}$ Var data assimilation, twin experiments were carried out with the dynamical core of the new FSU GSM consisting of a T126L14 global spectral model in a MPI parallel environment. Results and qualitative aspects are presented for incomplete data in the spatial dimension and for incomplete data in time, with and without inclusion of the background term into the cost functional. The importance of the background estimate on the $4 \mathrm{D}$-Var analysis in the presence of small Gaussian errors in incomplete data is also investigated.
\end{abstract}

Keywords: Data assimilation, incomplete observations, background error.

\section{Introduction}

A major issue in data assimilation is that the observing system providing full coverage, i.e., satellites rely on tuning procedures based on the radiosonde observing network and therefore are not well tuned over regions where the radiosonde network is sparse. In the southern hemisphere and tropics where most of the surface is covered by oceans, observations are sparse lacking density and uniformity of conventional wind-profile data coverage distribution available in Northern hemisphere.

In this paper, a new MPI-based parallel version of FSU global spectral model and its adjoint is used to study the impact of incomplete observations in space and time dimension on variational data assimilation. The impact of inclusion of a background error covariance term in the 4-D Var data assimilation cost functional formulation is analyzed. It is crucial for the performance of the analysis system to include the background term to ensure that observations produce statistically consistent increments for model variables that are both smooth and balanced. When data sparse areas or data void areas are present, the background propagates information from observations at earlier times into the data voids. 
This means that correlations in background error covariance matrix $\mathbf{B}$ will perform spatial spreading of information from observation points to a finite domain surrounding them.

Measurement of background error covariances has been addressed in the work of Hollingsworth and Lonnberg [2, Parrish and Derber 7], and Ingleby [3, to name a few. Since the background error covariance matrix is of huge dimensions, efficient ways to estimate it need to be derived. The inverse covariance matrix of $\mathbf{B}$ is represented via control variable transforms in order to obtain a simplification. See for instance Lorenc et al. [6].

The structure of this paper is as follows. In section 2, we briefly present a basic description of the FSU Global Spectral Model and its implementation. The formulation of the $4 \mathrm{D}$ variational data assimilation problem and the specification of the background error covariance matrix are discussed in section 3 . In section 4 numerical results with incomplete observations in both spatial and temporal domains are described in a twin experiments setup. Assimilation of sparse noisy data is also discussed. Summary and conclusions are presented in section 5 .

\section{Short Description of the FSU GSM}

The FSU GSM is a spectral global hydrostatic primitive equation model. The prognostic variables are vorticity, divergence, virtual temperature, moisture and logarithm of surface pressure. The model uses the spectral technique in the horizontal direction, and second order finite difference in the vertical. For details we refer to Krishnamurti et al. 44. The wave number truncation used in the numerical experiments is T126 for real time forecasts. Higher resolutions may be used for research purposes. A $\sigma$ coordinate system with $14 \sigma$-levels is used in the vertical. The model physics include long and shortwave radiation, boundary layer processes, large scale precipitation and shallow and deep cumulus convection.

\section{The Background Error in 4D-Var Data Assimilation}

One of the important issues in variational data assimilation is the specification of the background error covariance matrix. In recent years, a number of research efforts were dedicated to the study of what is known as the background error term denoted by $J_{b}$ and usually included in the definition of the basic cost function

$$
J_{b}\left(\mathbf{X}_{\mathbf{0}}\right)=\frac{1}{2}\left(\mathbf{X}_{\mathbf{0}}-\mathbf{X}_{b}\right)^{T} \mathbf{B}^{-1}\left(\mathbf{X}_{\mathbf{0}}-\mathbf{X}_{b}\right) .
$$

In the equation above $\mathbf{X}_{\mathbf{0}}-\mathbf{X}_{\mathbf{b}}$ represents the departures of the model variables at start of the analysis from the background field $\mathbf{X}_{\mathbf{b}} ; \mathbf{B}$ is an approximation to the covariance matrix of background error.

In this case, the cost function $J$ assumes the form

$$
J\left(\mathbf{X}_{\mathbf{0}}\right)=J_{b}\left(\mathbf{X}_{\mathbf{0}}\right)+J_{o}\left(\mathbf{X}_{\mathbf{0}}\right)
$$


where $J_{o}\left(\mathbf{X}_{\mathbf{0}}\right)$ is the distance to observations of a forecast initiated from $\mathbf{X}_{\mathbf{0}}$. With the inclusion of background error term, we can prove the uniqueness of the solution of the minimization process with incomplete observations for the linear case [8]. For the nonlinear case, uniqueness may be guaranteed under restricted conditions only.

\subsection{Formulation of the Background Error Covariance}

The background error covariance matrix is of rather huge dimension, its typical size being of $10^{6} \times 10^{6}$ elements. This is not tractable either from viewpoint of storage requirements or from that of available statistical information.

To avoid calculating the inverse of $\mathbf{B}$, we decided to proceed with a simple form of $\mathbf{B}^{-1}$ by taking $\mathbf{B}=\mathbf{D}^{\frac{1}{2}} \mathbf{C} \mathbf{D}^{\frac{1}{2}}$, where $\mathbf{C}$ was taken to vary only along the horizontal dimensions and $\mathbf{D}$ is a diagonal matrix. We defined

$$
\mathbf{C}^{-1} \approx w_{0} I+w_{2} \nabla^{2} \nabla^{2}
$$

where $w_{0}$ and $w_{2}$ are chosen such that the power spectrum is similar to that of the Gaussian correlation function (see Daley [1]):

$$
\mathbf{C}_{i j}=e^{-\frac{1}{2}\left(\frac{x_{i}-x_{j}}{l}\right)^{2}}
$$

where $x_{i}-x_{j}$ is the distance between grid points and $l$ is the correlation length scale.

The variance matrix $D$ was chosen to vary in the vertical such that its inverse diminished in the upper levels (M. Zupanski, personal communication).

$$
d^{\frac{1}{2}}=\alpha e^{-\beta\left(\left(p-p_{r e f}\right) / 1000 .\right)^{2}}
$$

where $d^{\frac{1}{2}}$ represents the square root of the diagonal element of $D$, which varies only along the vertical coordinate, $p$ is the pressure and $p_{\text {ref }}$ is reference pressure which taken the values from $100 \mathrm{hPa}$ to $250 \mathrm{hPa}$ according to different variables, so the the inverse of the variance will diminish around the upper reference pressure level. $\alpha$ and $\beta$ are used to adjust the distribution of $d^{\frac{1}{2}}$ along vertical coordinate.

\section{Numerical Experiments with Incomplete Observations}

Twin experiments were carried out using the dynamical core of the FSU GSM with complete observations which served as the control run. The length of data assimilation window was set to 6 hours from 06UTC Oct. 2, 2002 to 12UTC Oct. 2,2002 .

The observation data were generated by integrating the forward model 6 hours from an initialized ECMWF analysis at 06UTC Oct. 2, 2002 (thereafter referred to as unperturbed initial data), and were available at every timestep and at each Gaussian grid point. The initial guess of the initial condition was 
taken from the initialized ECMWF analysis at 00UTC Oct. 2, 2002 (thereafter refer to perturbed initial data), which is 6 hours prior to the initial time level.

A suitable choice of the background state $\mathbf{X}_{b}$ is important, since it will strongly affect retrieved initial data. In our experiments, the data were generated by integrating the forward model 6 hours prior to the initial time 00UTC Oct. 2, 2002, arriving at same initial time as the start time of the data assimilation window (06UTC Oct. 2, 2002). The limited-memory quasi-Newton method of Liu and Nocedal [5] (L-BFGS) was used to carry out the unconstrained minimization process to obtain the optimal initial data. To simulate the case of incomplete observations, we reduced the number of observations available in the space dimension to every 2, 4 or 8 grid points, respectively. In order to investigate impact of incomplete observations over data void areas, we carried out an experiment where observations over all grid points located over oceans of the Southern hemisphere were missing. In another experiment we reduced the observations in the time dimension to be available only every 2,4 or 8 timesteps, respectively. The impact of the background term in the retrieval of initial conditions is further analyzed in experiments where small random, Gaussian, noise is added to the incomplete observational data.

\subsection{D-Var Assimilation Experiments Without Background Term}

Figure1 provides the rms errors of height field at $500 \mathrm{hPa}$, calculated between the fields of model generated observations and the fields obtained by integrating the optimized initial data with incomplete observation after 40 function evaluations (left) and after 100 function evaluations (right). It shows that for incomplete observations in space dimension, the error reduction obtained by the minimization process for incomplete observations depends on density of observations in space dimension.

For observations available every 2 grid points, although the decrease rate of the cost function is slowed down, it can still retrieve the initial data to a certain degree, while for observations available only at every 4 or 8 grid points, the errors increase to a higher degree. The sparser the density of the observations, the larger the departure from observations obtained with minimization of incomplete observations. This issue becomes evident for the data-void area experiment in which case the retrieved initial data were erroneous (i.e. rms difference was quite large). For incomplete observations in the time dimension there were only minor differences in the retrieved initial data for the different cases considered.

\subsection{Impact of the Background Error Term on 4D-Var}

Since the background error term provides information related to the initial data, we carried out a number of experiments with incomplete observations in the space dimension with a background error term $J_{b}$ included in the cost function. The results show that the behavior of the minimization process when background covariance term is included in the cost functional is quite different from the one without its inclusion. Figure 2 (left) shows the rms errors of height field at 500 

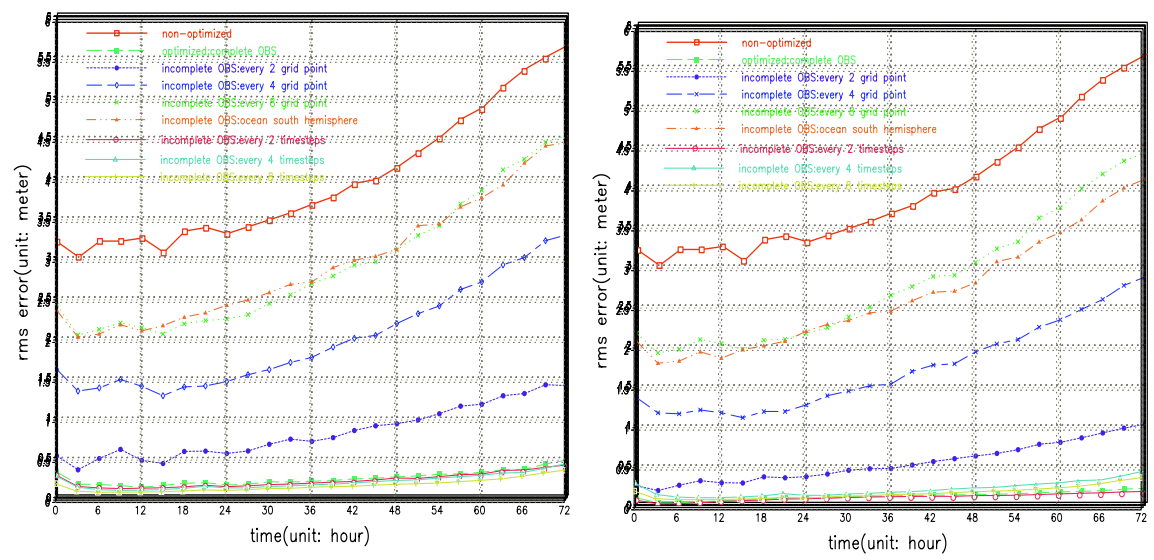

Fig. 1. Time evolution of rms of the height field at $500 \mathrm{hPa}$ after 40 function evaluations (left) and after 100 function evaluations (right) for different cases of incomplete observations without inclusion of background error term, red( $\square)$-non-optimized, i.e. result obtained by integrating perturbed initial condition, aqua (ם, long dash)-optimized by complete observations, dark purple( $\bullet$, short dash)-optimized by incomplete observations available every 2 grid points, dark blue $(\diamond$, long dash short dash)-optimized by incomplete observations available every 4 grid points, light green(x, dotted)-optimized by incomplete observations available every 8 grid points, orange $(\boldsymbol{\Lambda}$, dot dot dash)optimized by incomplete observations that data missing over south hemisphere oceans, magenta $(\circ$, solid)-optimized by incomplete observations available every 2 timesteps, light blue $(\triangle$, solid)-optimized by incomplete observations available every 4 timesteps, yellow(x, solid)-optimized by incomplete observations available every 8 timesteps

$\mathrm{hPa}$, calculated between the fields obtained by integrating the optimized initial data with complete observations and the fields obtained by integrating optimized initial data with incomplete observations after 40 function evaluations. It shows that for incomplete observations in the space dimension, like for the case of exclusion of background error term, the error reduction obtained by the minimization process with incomplete observations depends on the density of the observations in space dimension. We observe that the rms curves after 40 function evaluations exhibit 6- hour oscillations for both incomplete observations in space ( observations available every 2-gridpoints) as well as for incomplete observations in time. To assess whether this oscillation is due to the fact that inclusion of background error term altered rate of convergence of the minimization, we carried out an experiment where the rms curves were calculated after 100 function evaluations. The results are also shown in Fig. 2 (right) and we noticed that in this case the abovementioned oscillations vanished which points strongly to the fact that inclusion of background error term has altered structure of spectrum of Hessian of cost functional, thus changing convergence rate characteristics of the minimization. 

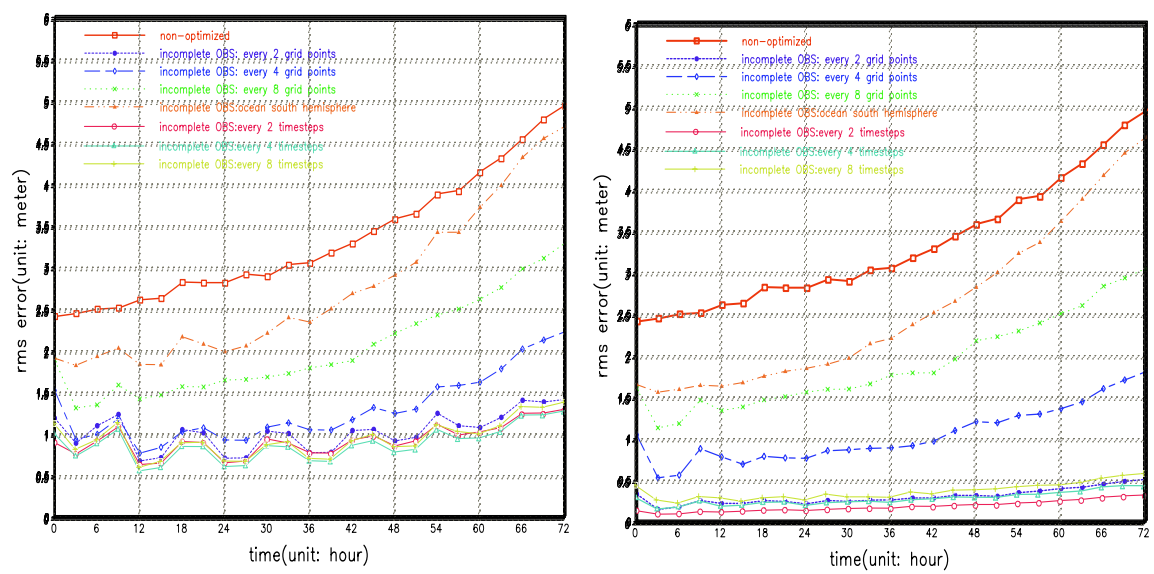

Fig. 2. Time evolution of rms of the height field at $500 \mathrm{hPa}$ after 40 function evaluations (left) and after 100 function evaluation (right) for different cases of incomplete observations with inclusion of background error term, $\operatorname{red}(\square)$-non-optimized, i.e. result obtained by integrating the perturbed initial condition, dark purple $(\bullet$, short dash)-optimized by incomplete observations available every 2 grid points, dark blue $(\diamond$, long dash short dash)-optimized by incomplete observations available every 4 grid points, light green(x, dotted)-optimized by incomplete observations available every 8 grid points, orange $(\boldsymbol{\Lambda}$, dot dot dash)-optimized by incomplete observations where data is missing over south hemisphere oceans, magenta $(\circ$, solid $)$-optimized by incomplete observations available every 2 timesteps, light blue $(\Delta$, solid)-optimized by incomplete observations available every 4 timesteps, yellow(x, solid)-optimized by incomplete observations available every 8 timesteps

\subsection{The Impact of the Background Term in the Presence of Errors in Incomplete Observations}

In this section numerical experiments are used to investigate the impact of the background term on the retrieval of the initial conditions when a small Gaussian noise of up to $0.1 \%$ is added to the incomplete observations. For briefness, we discuss only two representative cases: first, perturbed observations are incomplete in space, available on a sparse subgrid at every 8 grid points for each horizontal level; second, perturbed observations are incomplete in time, available every 8 time steps (each hour since the integration time step is $\Delta t=450 \mathrm{~s}$ ). For each case two data assimilation experiments are set up: one without the background term and one with the background term included in the cost functional. The ECMWF analysis at 06UTC Oct. 2, 2002 is used as reference initial state in the qualitative analysis of the results. For computational considerations, we restricted the minimization process to 25 iterations or 30 function evaluations, whichever limit is reached first. 
Incomplete Observations in Space. In a first experiment, we consider the assimilation of noisy data, sparse in the spatial dimension, in the absence of the background term. The errors in the retrieved initial conditions for the $500 \mathrm{hPa}$ height field ranged from $-8 \mathrm{~m}$ to $10 \mathrm{~m}$ and we noticed that the analysis errors were distributed throughout the spatial domain with no particular structure and with magnitude about twice as large as the errors in observations. When the background term was included into the cost functional we noticed that the distance to observations increases during the first 24 iterations and the analysis was significantly closer to the background. Therefore we expect a slow assimilation process that may benefit from a better scaling between $J_{b}$ and $J_{o}$. The errors in the retrieved initial conditions for the $500 \mathrm{hPa}$ height field typically ranged from $-10 \mathrm{~m}$ to $10 \mathrm{~m}$.

Incomplete Observations in Time. Assimilation of the noisy data, incomplete in time, and without background term provided improved results as compared to the noisy data, incomplete in space experiment. In this case, the errors in the retrieved initial conditions for the $500 \mathrm{hPa}$ height field ranged from $-5 \mathrm{~m}$ to $4 \mathrm{~m}$. The experiment with the background term included into the cost functional provided an analysis closer to both background term and observations. Errors in the retrieved initial conditions for the $500 \mathrm{hPa}$ height field typically ranged from $-10 \mathrm{~m}$ to $10 \mathrm{~m}$.

\section{$5 \quad$ Summary and Conclusions}

We analyzed the impact of inclusion of the background error term for incomplete observations in either space or time in the framework of 4-D Var data assimilation with the FSU GSM and its adjoint model. First we carried out the experiments on the impact of incomplete observations in absence of the background error term for 4-D Var data assimilation with the FSU GSM. Results show that for incomplete observations in space dimension, the minimization process fails to successfully retrieve the initial data, while for incomplete observations in time dimension, the minimization process can retrieve the initial data. Then we carried out a series of experiments on the impact of background term on incomplete observations for 4-D Var data assimilation with the FSU GSM. For the sake of simplification, we calculated the inverse of the background covariance matrix $\mathbf{B}^{-1}$ directly by using a diffusion operator. This avoided the calculation of the inverse of a huge dimension matrix.

Results obtained show that, inclusion of the background error term had a positive impact on convergence of minimization for incomplete observations in space dimension. The sparser the incomplete observations in space dimension, the stronger was the impact of the background error term. However for the case of a data void over the southern hemisphere oceans, the convergence of minimization was observed to be slowed-down.

In contrast to the case of incomplete observations in the space dimension, the background error term had a negative impact on the convergence of minimiza- 
tion for incomplete observations in time dimension. The sparser the incomplete observations in time dimension, the larger the negative impact of the background error term. The time evolution of the rms error of the height field at $500 \mathrm{hPa}$ for a 72-hours forecast for different cases of incomplete observations with and without inclusion of the background error term were discussed.

Numerical experiments with small noise added to the incomplete observations were also considered. In the absence of the background term, we noticed that errors in incomplete observations in space resulted in larger errors in the analysis estimate, whereas errors in incomplete observations in time resulted in errors of similar magnitude in the analysis estimate. When the background term was included, assimilation of noisy incomplete data in space resulted into a slow optimization process, with the analysis state close to the background estimate and further from data. By contrast, the analysis state provided by the assimilation of incomplete data in time provided an analysis closer to both background and observations.

These experiments also indicate that in the case of incomplete observations the specification of the background estimate becomes of crucial importance to the analysis. Extension of this study to a full physics version with realistic observations should provide additional insight about the role played by the background error in 4-D Var with incomplete observations.

\section{Acknowledgements}

This work was funded by NSF Grant ATM-0201808. The authors would like to thank Dr. Linda Peng the grant manager. We would like to thank Dr. Milija Zupanski for his helpful and insightful advice and his generous sharing of his code.

\section{References}

1. Daley, R.: Atmospheric Data Analysis. Cambridge University Press, New York (1991)

2. Hollingsworth, A. and Lönnberg, P.: The statistical structure of short-range forecast errors as determined from radiosonde data. Part I:the wind field. Tellus 38A (1986) 111-136.

3. Ingleby, N.B.: The statistical structure of forecast errors and its representation in the Met Office global 3D variational data assimilation scheme, Q.J.R. Meteorol. Soc. 127 (2001) 209-231.

4. Krishnamurti T.N., Bedi, H.S. and Hardiker, V.M.: An introduction to global spectral modeling, Oxford University Press (1998)

5. Liu, D.C., and Nocedal, J.: On the limited memory BFGS method for large scale optimization. Math. Programm. 45 (1989) 503-528.

6. Lorenc, A.C. and Coauthors: The Met Office global 3D variational data assimilation scheme. Q. J. R. Meteorol. Soc. 126 (2000) 2991-3012.

7. Parrish, D.F. and Derber, J.: The national meteorological center's spectral statistical-interpolation analysis system. Mon. Wea. Rev. 120 (1992) 1747-1763.

8. Zou, X., Navon, I.M. and Le-Dimet, F.X.: Incomplete observations and control of gravity waves in variational data assimilation. Tellus 44A (1992) 273-296. 